\title{
On the Relation Between Oxide Ridge Evolution and Alloy Surface Grain Boundary Disorientation in Fe-22 wt \% Cr Alloys
}

\author{
Jingxi Zhu, ${ }^{\text {a,b,z }}$ Laura M. Fernandez Diaz, ${ }^{\text {a,b }}$ Gordon R. Holcomb, ${ }^{\text {c,* }}$

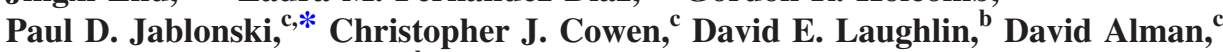 \\ and Seetharaman Sridhar ${ }^{a, b}$ \\ ${ }^{a}$ National Energy Technology Laboratory, Pittsburgh, Pennsylvania 15236, USA \\ ${ }^{b}$ Department of Materials Sciences and Engineering, Carnegie Mellon University, Pittsburgh, \\ Pennsylvania 15213, USA \\ ${ }^{c}$ National Energy Technology Laboratory, Albany, Oregon 97321-2198, USA
}

Oxide ridges formed during the transient stage oxidation of the scale evolution in iron alloys containing $22 \mathrm{wt} \% \mathrm{Cr}$ that were held at $800^{\circ} \mathrm{C}$ in dry air. The surface oxidation process was imaged in situ through a confocal scanning laser microscope, and the results were correlated with postexperiment characterization through scanning electron microscopy and the DualBeam system (focus ion beam and electron beam) analysis combined with three-dimensional reconstruction. The oxide ridges that formed on top of the Cr oxide scale overlapped the intersections of the underlying alloy grain boundaries with the Cr oxide scale. Ridges were generally very small on grain boundaries with disorientation angles of less than $15^{\circ}$, and it was suggested that the boundaries of the surface grains in the alloy may serve as bottlenecks for the transport of scale-forming elements. The effects of La (120 and $290 \mathrm{ppm})$ and Ce (270 and $610 \mathrm{ppm})$ additions during melt-stage processing were also investigated.

(c) 2010 The Electrochemical Society. [DOI: 10.1149/1.3308571] All rights reserved.

Manuscript submitted August 25, 2009; revised manuscript received November 27, 2009. Published March 26, 2010.

Solid oxide fuel cells (SOFCs) are considered to be promising energy conversion devices that can electrochemically convert a range of fuels including syngas and methane into electricity. Among the different types of fuel cell designs, the flat plate (planar) design is now more favored due to the ease of processing and manufacturing the cell components. ${ }^{1}$ This planar design requires the use of interconnectors in the SOFC stack that provide mechanical support to the cell components as well as the electrical contact between single cells and that separate the fuel on the anode side from the oxidant on the cathode side. Ceramic oxides based on doped lanthanum chromite and on relatively expensive high $\mathrm{Cr}$ superalloys have been used as interconnector materials. ${ }^{2}$ However, lowering the operational temperature of SOFCs from around 1000 to $800^{\circ} \mathrm{C}$ offers the possibility of using less expensive alloys.

Because the scales formed at SOFC operating temperatures must be electrically conductive for interconnector applications, alumina or silica formers are not suitable. Fe-Cr-based alloys (less than 24 wt $\% \mathrm{Cr}$ ) are considered the favorable candidates for metallic interconnectors in planar cells ${ }^{2}$ because there is a good balance between the slow growth rate of the oxide scale and the electrical conductivity of the oxide scale. Moreover, they have an appropriate thermal expansion coefficient that closely matches that of the other components of the cell. ${ }^{2,3}$ Finally, they also offer good mechanical support for the entire cell structure. A major problem with metallic interconnectors, such as those based on $\mathrm{Fe}-\mathrm{Cr}$ alloys, is their reactivity with the anode and/or cathode side service environments at operating temperatures. ${ }^{2}$ The resulting high temperature corrosion or oxidation can lead to the failure of the cell. Also, volatile $\mathrm{CrO}_{3}$ and/or $\mathrm{CrO}_{2}(\mathrm{OH})_{2}$ can form at the oxide scale/oxidant interface, migrate to the cathode/electrolyte interface, and form chromia deposits, which can poison the cathode reaction and reduce SOFC efficiencies. ${ }^{2}$

The oxidation of metals and alloys takes place by outward diffusion of metal and/or inward diffusion of oxygen through the oxide scale. ${ }^{4}$ The outward diffusion of metal normally results in the formation of an external scale that grows at the scale/gas interface as well as in the formation of voids and cavities at the scale/alloy interface that can lead to scale spallation. ${ }^{2}$ In many cases, it is the properties of this external scale that determine the oxidation resistance of the alloy. Many studies have been carried out to understand the high temperature oxidation mechanism and to improve the oxi-

* Electrochemical Society Active Member

z E-mail: jingxiz@andrew.cmu.edu dation resistance of $\mathrm{Fe}-\mathrm{Cr}$ alloys. It has been reported that when reactive elements are added to $\mathrm{Fe}-\mathrm{Cr}$ alloys, the scale no longer grows by outward diffusion of $\mathrm{Cr}$ but by inward diffusion of oxygen, which significantly improves the scale adhesion. ${ }^{2}$ Hojda et al. ${ }^{5}$ developed a commercial alloy, Crofer 22 , by adding $\mathrm{Mn}, \mathrm{Ti}$, and $\mathrm{La}$ to a $\mathrm{Fe}-20-24$ wt $\% \mathrm{Cr}$ base alloy.

Compared to steady-state oxidation, transient oxidation has been much less studied. However, some knowledge on the growth mechanism of the scale formed in the initial stage of oxidation is necessary because the nature of this transient scale, such as its spatial distribution, morphology, composition, structure, and/or any pertinent features, can influence the nature of the steady-state scale. ${ }^{6}$

In an earlier paper, the authors observed scale ridge formation at the very early stage of oxidation, ${ }^{7}$ and the scale ridge formation related to the alloy grain boundaries (GBs) beneath. Fast-path diffusion through GBs can be categorized into three kinetic types, A, B, and $\mathrm{C}$, according to Harrison. ${ }^{8}$ For type A, the material acts as a uniform medium for diffusion with an effective diffusion coefficient. Type $\mathrm{C}$ represents situations in which diffusion occurs exclusively through the GB, and any case between the two extremes is type B, in which volume diffusion and GB diffusion simultaneously occur. While the presence of ridges coinciding with the underlying alloy GBs suggests that the kinetics are of either type C or more likely B (as shown below), it was not established in the earlier study ${ }^{7}$ if there was a correlation between the GB characteristics and the presence or size of ridges. The purpose of the present investigation is to examine the relationships between fast-path diffusion during transient oxidation with respect to the geometry of alloy GBs by analyzing the scale ridge features and the underlying alloy GB characteristics. The alloys considered in this study are similar to a Crofer 22 alloy with $22 \mathrm{wt} \% \mathrm{Cr}$, in which Ce was also added along with La. The possible role(s) of rare-earth (RE) additions are also investigated.

\section{Experimental}

Alloy studied.- All the test samples were manufactured at the National Energy Technology Laboratory (NETL) of the U.S. Department of Energy. An Fe-22 wt \% Cr ferritic stainless steel was selected as the base material and was produced in-house at the NETL's Albany, Oregon site. Vacuum induction melting was used to produce $7 \mathrm{~kg}$ ingots from high purity starting elemental materials. After casting, the alloys were reduced to sheet by hot working and cold rolling.

Mish-metal additions of RE elements were added to the melt 


\begin{tabular}{|c|c|c|c|c|c|c|}
\hline & $\mathrm{Cr}$ & $\mathrm{C}$ & $\mathrm{Mn}$ & $\mathrm{Ti}$ & $\mathrm{La}$ & $\mathrm{Ce}$ \\
\hline $\mathrm{F}_{1}$ & 22.18 & 0.002 & 0.43 & 0.1 & N/A & N/A \\
\hline $\mathrm{F}_{2}$ & 22.12 & 0.005 & 0.55 & 0.072 & 0.012 & 0.027 \\
\hline $\mathrm{F}_{3}$ & 22.19 & 0.019 & 0.56 & 0.077 & 0.029 & 0.061 \\
\hline
\end{tabular}

before the casting of the alloys. Glow discharge mass spectroscopy was used to analyze the RE elemental content of the alloys, as summarized in Table I.

The samples for the oxidation study were made with dimensions of $5 \times 5 \times 1 \mathrm{~mm}$, ground with $\mathrm{SiC}$ paper to 1200 grit, and then fine-polished with 6,3 , and $1 \mu \mathrm{m}$ diamond suspensions. These polished samples were subsequently cleaned with soapy water and then ultrasonically cleaned in acetone and ethanol. The processing and the experiments carried out on the two groups of samples used in the current study are summarized in Table II.

High temperature oxidation.- Polished samples were oxidized in a hot stage attached to a confocal scanning laser microscope (CSLM). The hot stage was composed of a gold-image furnace and a halogen lamp, as shown in Fig. 1. The furnace chamber was ellipsoidal so that the radiation from the halogen lamp (located at the lower focus of the ellipsoid) was reflected by the gold furnace wall and was concentrated on the sample placed in the crucible (upper focus of the ellipsoid). With this design, the furnace was able to achieve a high heating rate; for example, heating from room temperature to $800^{\circ} \mathrm{C}$ took $45 \mathrm{~s}$. This was very important for studying the transient stage oxidation. Moreover, the microscope was equipped with a helium laser source and with confocal optics that allowed a direct observation of the material's microstructure at high temperature due to a much higher intensity of the laser radiation than the thermal radiation from the sample surface. A thermocouple was placed near the sample surface to measure the temperature. The measured temperature was used to control the heating intensity, and the temperature profile of the oxidation experiment was always programmed before oxidation. To ensure an accurate temperature measurement during heating, the readings of the thermocouple were calibrated by heating pure metals of known melting temperatures. A linear relationship between the program and the actual temperature was obtained after calibration, with the $R^{2}$ (square of correlation coefficient) value greater than 0.99 . Before every oxidation experiment, the furnace chamber was evacuated and refilled with dry air, which was subsequently allowed to flow for $5 \mathrm{~min}$. This evacuation and refilling step was repeated three times. The flow rate of dry air was controlled at $500 \mathrm{~mL} / \mathrm{min}$ during high temperature exposure. The required heating time from room temperature to $800^{\circ} \mathrm{C}$ was 45 $\mathrm{s}$, and the samples were maintained at this temperature for $15 \mathrm{~min}$, after which they were cooled to room temperature in another $45 \mathrm{~s}$. All the samples examined in this study were oxidized under the aforementioned conditions. The oxidized samples were then used for scale characterization.
Characterization.-SEM and focused ion beam.- The oxidized surfaces of group I samples, $F_{1}, F_{2}$, and $F_{3}$, were examined by a scanning electron microscope (SEM, Philips XL 30, field-emission $\mathrm{SEM})$. Following the plane-view characterization of the scales, cross-sectional characterization was performed. Cross sections were obtained by milling a rectangular hole (ca. $7 \times 3 \mu \mathrm{m})$ into the surface with a Nova 600 DualBeam system. The region of interest to be cross sectioned (approximately $20 \mu \mathrm{m}$ ) was covered with a $1 \mu \mathrm{m}$ thick Pt layer to avoid damage while milling. The surface was cross sectioned to a depth of $4-5 \mu \mathrm{m}$ using a $30 \mathrm{kV} \mathrm{Ga}{ }^{+}$ion beam with a current of ca. $0.5 \mathrm{nA}$.

$3 D$ reconstruction from automated serial cross sectioning FIB.- A randomly chosen area of scale and underlying alloy grains was reconstructed with serial-sectional secondary electron images from sample $\mathrm{F}_{2}$ obtained by focused ion beam (FIB). A cross section was created first, and then the material at the edge of this cross section was removed slice by slice, with a slice thickness of $0.48 \mu \mathrm{m}$. Sequential SEM images were then taken after every slice was removed. In this way, a series of 100 images was obtained, from which the scale layer and underlying alloy grains were reconstructed in a three-dimensional (3D) space using the software Amira 3.1.

Orientation imaging and observation of scale ridges.-Electron backscattered diffraction (EBSD) was used to generate orientation maps of the polycrystalline alloy surfaces before oxidation on a $F_{2}$ specimen of group II. Because the alloys used in this study were ferritic alloys that lack an allotropic phase transformation, a requirement to ensure that GB migration and growth did not occur during the oxidation experiments existed. To stabilize the alloy GBs and to release residual stress left from cold work, the $\mathrm{F}_{2}$ specimen was annealed before orientation mapping for $1 \mathrm{~min}$ at $1500^{\circ} \mathrm{C}$ followed by $45 \mathrm{~min}$ at $800^{\circ} \mathrm{C}$. The specimen was then mechanically polished and electropolished before EBSD mapping. Upon completion of the EBSD mapping, the specimen was subsequently oxidized under the conditions described in the High temperature oxidation section. The formed scale ridges were observed with SEM and were compared to the GB map obtained before oxidation. The flowchart in Fig. 2 shows the several steps involved in the orientation imaging and in the observation of the scale ridge size performed.

The EBSD map was collected using an EDAX-TSL Hikari high speed detector controlled by TSL OIM data collection v5.21 software. The backscattered diffraction patterns were collected using an accelerating voltage of $25 \mathrm{kV}$ at $9.8 \mathrm{nA}$. Orientation data were collected with a resolution of $10 \mu \mathrm{m}$. The orientation of the crystal

Table II. The processing routes and experiments carried out on the two groups of samples used in the current study.

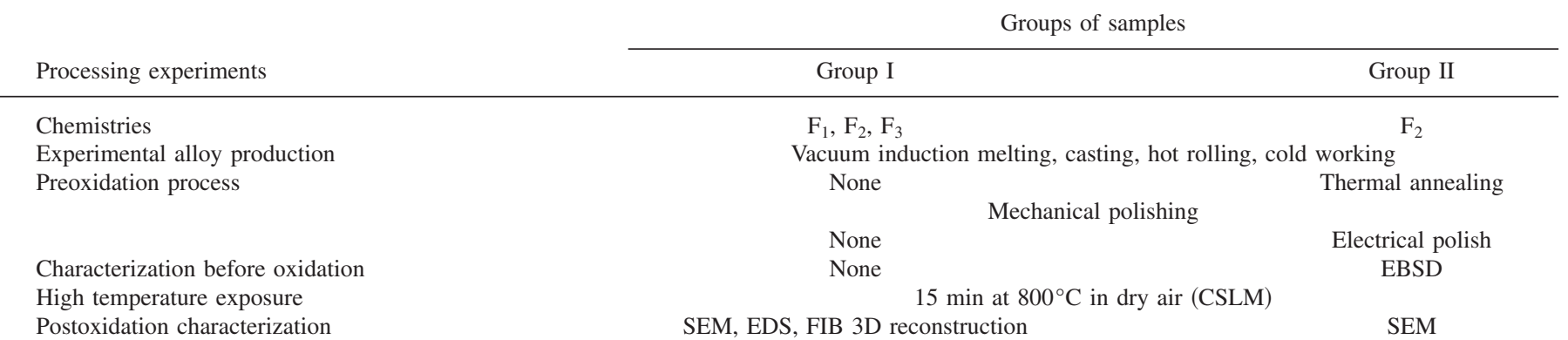




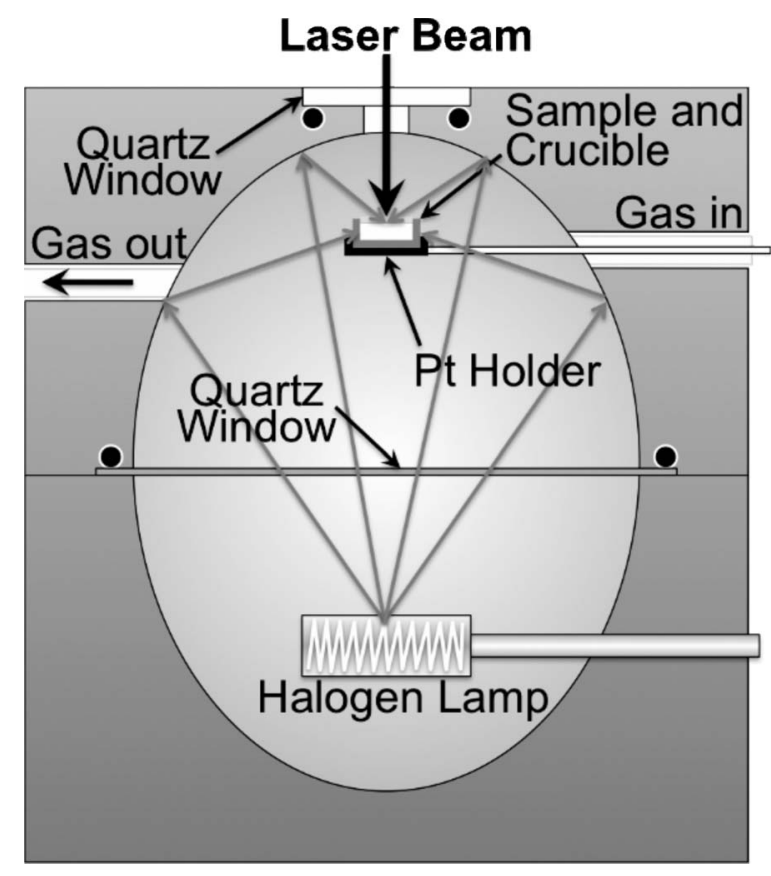

Figure 1. Schematic of the gold-image furnace.

lattice of each grain with respect to an arbitrary reference frame was represented in the form of the three "Euler angles."

EBSD mapping data analysis. - The EBSD map was cropped, and poorly indexed points were removed using the "grain dilation" procedure in the TSL OIM analysis software with a minimum disorientation angle of $5^{\circ}$. After cleanup, a "single orientation" was assigned to each grain. The GBs were reconstructed by fitting line segments between triple points on a hexagonal grid such that the boundary segments did not deviate from the true boundary position by more than 2 pixels. ${ }^{12}$ Next, the disorientation angle between two neighboring grains was calculated for each GB segment with a low angle cutoff of $5^{\circ}$. TSL OIM Analysis software expressed the "misorientation" of two grains in an angle-axis pair convention. The software

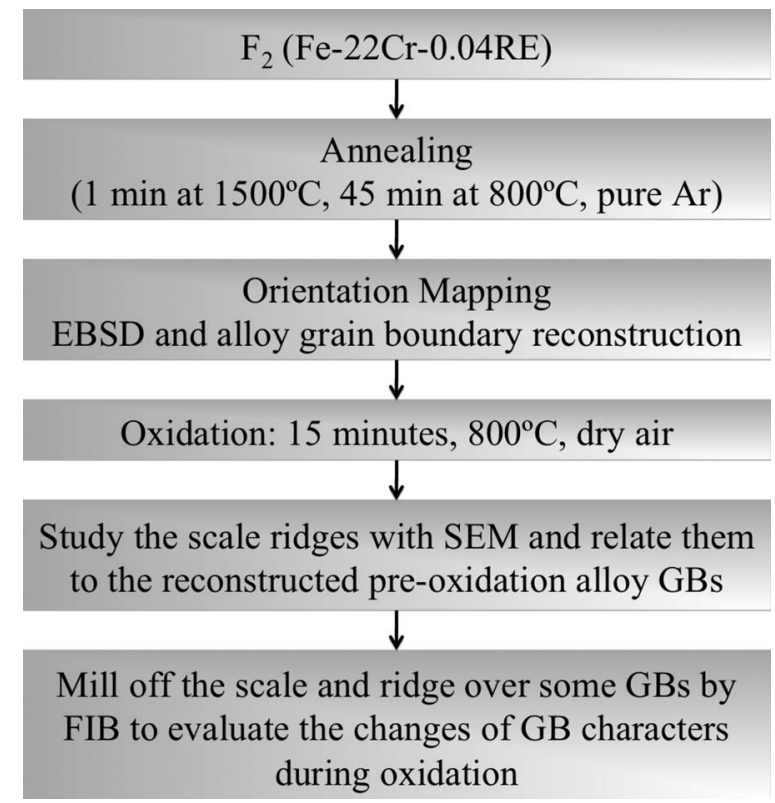

Figure 2. Flowchart of the investigation of the relationship between scale ridge size and alloy $\mathrm{GB}$. read the orientation information of the two neighboring grains and returned the rotation angle that has the smallest value of misorientation angle possible. This minimum rotation angle is known as the disorientation angle.

Finally, to establish a reliable relationship between the scale ridge size and the misorientation of the alloy GBs, it was necessary to evaluate the changes in alloy GB characteristics after the 15 min oxidation exposure.

The scale and scale ridges of a small area of several square micrometers across two grains were first milled away by FIB. Then, the alloy grains under the scale were exposed and scanned with the OIM system, and GB disorientations were later calculated from the EBSD maps. This milling and EBSD step was repeated for 24 different sites to include different types of GBs.

\section{Results}

From the real-time recording of the oxidation process, oxide nodules were seen to grow quite fast from the entire surface when the temperature exceeded $650^{\circ} \mathrm{C}$. This was expected to be a gas-phase mass-transfer process that would result in a linear oxidation rate. ${ }^{4} \mathrm{As}$ the sample was heated, the surface became covered by a scale. The ridges became clearly observable a few seconds after the temperature reached $800^{\circ} \mathrm{C}$, followed by the growth of the scale on top of the grain interiors. In Fig. 3, oxide nodules and traces of scale ridges are visible. The most obvious evolution of the scale ridges on the sample surface over time was the development in intensity of the contrast, which was represented as the darkening of the ridges. The CSLM was able to discriminate the light reflected from points on and away from the focal plane and to produce a white and black contrast. As the ridges grew larger, they rose above the surface and became successively darker in the confocal image.

An earlier study ${ }^{13}$ on scale ridge formation proposed three types of ridge growth kinetics. All three types were observed in the current study. However, all three types of growth kinetics were observed on both the straight GBs and curved ones unlike what was observed in the work reported by Thorning and Sridhar, ${ }^{13}$ where certain growth kinetics were associated with either straight or curved boundaries.

The postexperiment characterization of the sample surface can be seen in Fig. 4. Ridges can be found all over the surface, as shown in Fig. 4a and c, and details of ridges (indicated by the black dotted line) can be seen in Fig. 4b and d. The ridges can barely be seen on the $\mathrm{F}_{3}$ sample at the same magnification as in Fig. $4 \mathrm{a}$ and b, which is shown in Fig. 4e, and the details of its ridges are shown in Fig. 4f. From the results in Fig. 4, it seems that the formation of ridges during oxidation occurred on all of the samples of alloys $F_{1}, F_{2}$, and $\mathrm{F}_{3}$, but the ridges are generally smaller on the $\mathrm{F}_{3}$ sample when compared to $F_{1}$ and $F_{2}$, and the $F_{3}$ sample contained the largest amount of RE elements.

Energy dispersive X-ray analysis (EDS) results (Fig. 5) indicated that the oxide nodules constituting the ridges were primarily chromium oxide, but when compared to the nodules in the grain interior, there was often an increased amount of $\mathrm{Mn}$ and sometimes of $\mathrm{Ti}$ oxides in the ridges.

Figure 6 shows the cross sections of typical ridges on the surfaces of alloys $F_{1}, F_{2}$, and $F_{3}$ obtained with FIB. It can be seen from these cross-section images that ridges in all three cases are possibly located where the underlying metal GBs intersect the alloy/scale interface. Intergranular oxidation can also be observed in Fig. 6 (where the arrows are pointing). The fact that the ridges are rich in $\mathrm{Cr}$ and $\mathrm{Mn}$ suggests that the metal GBs may act as preferential diffusion paths for these elements. The width and height of the ridges decrease with increasing RE content in the alloy (which is consistent with plane view SEM observations shown in Fig. 4). This observation could be attributed to the GB diffusion of $\mathrm{Cr}$ or $\mathrm{Mn}$ being blocked progressively with the increase in RE content in the alloy.

To strengthen the observed relationship between ridges and GBs of the alloy, a random area of scale was reconstructed with serialsectional SEM images of sample $\mathrm{F}_{2}$ obtained through serial stepwise 


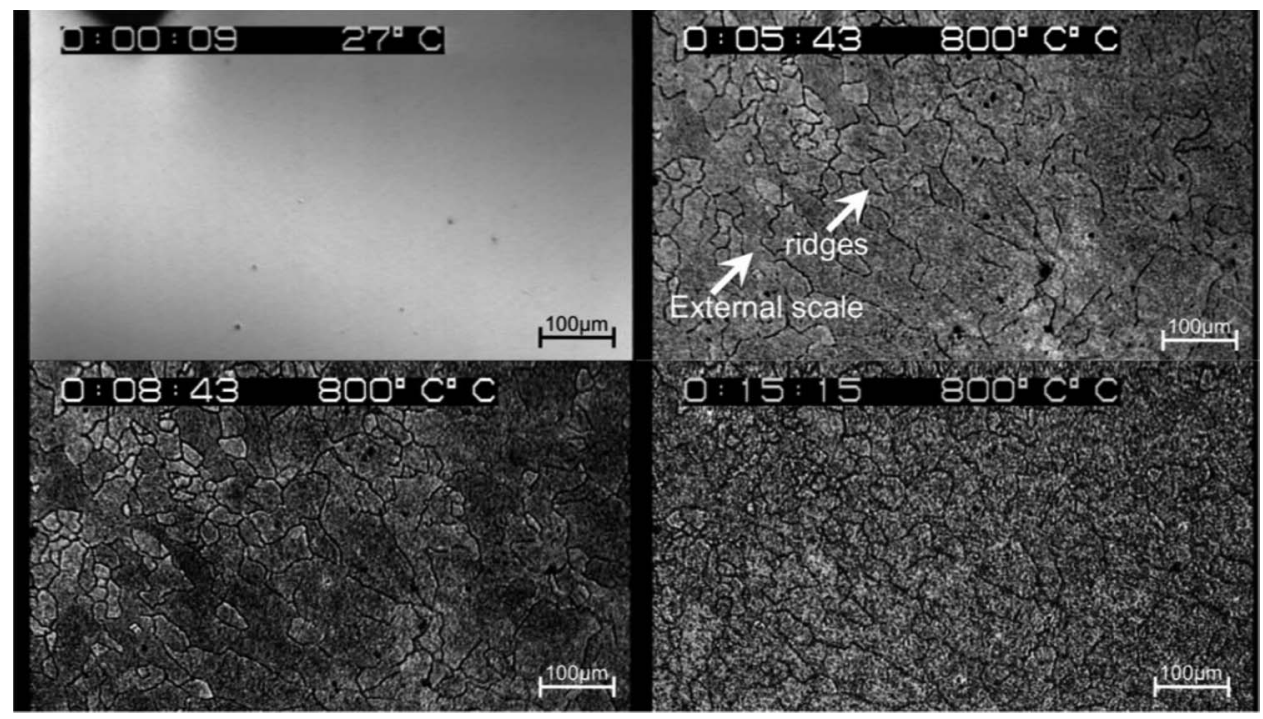

Figure 3. CSLM images of oxidation at $800^{\circ} \mathrm{C}$ extracted from real-time recordings. sectioning using FIB. The reconstructed scale layer is shown in Fig. 7. A triple junction of ridges can be seen on the scale layer. The reconstruction shows that the scale layer is made up of oxide nodules (as the one circled in Fig. 7), but only those places where the alloy GBs intersect the scale seem to have continuous ridges. The nodule, indicated by the circle, would appear as a ridge in a twodimensional cross section such as those in Fig. 6, but it can be seen
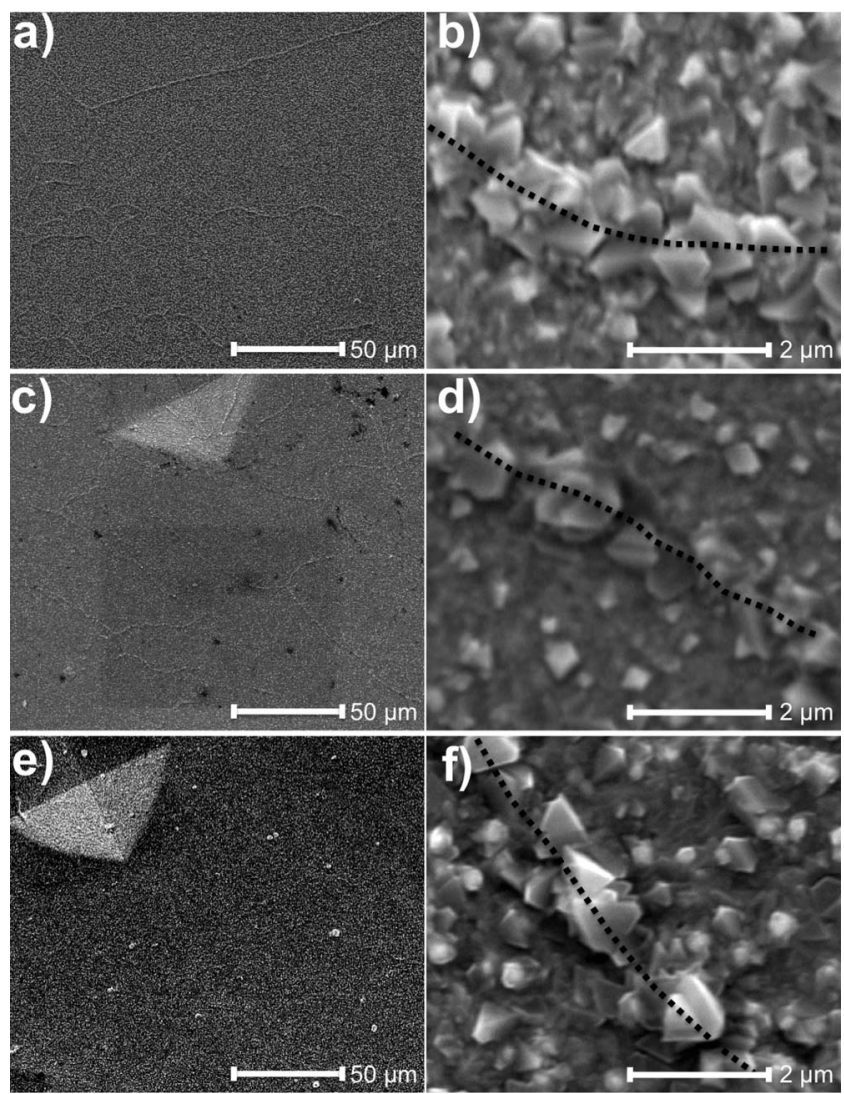

Figure 4. SEM secondary electron images of alloys $F_{1}$ and $F_{2}$ after a 15 min oxidation at $800^{\circ} \mathrm{C}$ in dry air. (a) General view of the surface of alloy $\mathrm{F}_{1}$. (b) Ridge formed on the scale of alloy $F_{1}$. (c) General view of the surface of alloy $F_{2}$. (d) Ridge formed on the scale of alloy $F_{2}$. (e) General view of the surface of alloy $F_{3}$. (f) Ridge formed on the scale of alloy $F_{3}$. in the $3 \mathrm{D}$ reconstruction that it does not have a GB associated with it in the underlying alloy and that it is clearly not part of a continuous ridge.

To further investigate the relationship between the alloy GB characteristics and the oxide ridges, the alloys were annealed in pure Ar before oxidation to obtain an equiaxed and larger grain structure that can be more readily quantified in terms of boundary types and characteristics. Figure 8a shows the EBSD mapping from the annealed sample $\mathrm{F}_{2}$, and the corresponding coloring of the grains based upon grain orientation can be found from the inverse pole figure of ferrite (Fig. 8b). The alloy GBs were reconstructed from the cleaned up EBSD map, as shown in Fig. 8c, in which gray indicates low disorientation angle $\left(<15^{\circ}\right)$ boundaries and black indicates boundaries with disorientation angles larger than $15^{\circ}$. It can be seen that after preoxidation annealing, the alloy grains have completely recrystallized and have clearly proceeded to the stage of grain coarsening. No cold worked texture remained. More than $86 \%$ of the total length of the GBs were large angle $\left(>15^{\circ}\right)$ boundaries. The GBs seemed quite stable, judging from dihedral angles at triple junctions and also from the GB curvature and were therefore suitable for the investigation of GB diffusion. ${ }^{14}$

A comparison of the GB characteristics measured before and after oxidation indicated that the disorientation angles in most of the cases varied within several degrees, which were acceptable system errors: All of the low angle boundaries examined stayed as low angle boundaries during oxidation; most coincident site lattice (CSL) boundaries (special boundaries) remained as CSL boundaries; and disorientation angles for high angle boundaries barely changed. Therefore, it was feasible to relate the scale ridge morphology obtained with SEM after oxidation to the GB characteristics measured before oxidation.

Figure 7 shows how intersections of GB and the oxide scale match with the locations of the oxide ridges, and Fig. 9 shows a surface view of the scale and scale ridges along with the preoxidized sample surface characterized by OIM at the same location of the oxidized sample. Scale ridges observed on this sample, again, matched well with alloy GBs, as shown in Fig. 9. By examining the ridges on the entire sample surface and by comparing them to the GB map, it can be stated that more than $95 \%$ of the GB length remained stationary during the oxidation. Only a few very small grains disappeared during oxidation, and the boundaries of these grains occupy only a small fraction of the total GB length.

Figure 9a is a plane-view SEM image of several scale ridges, which appear in a brighter contrast in secondary electron images. The two-headed arrow across each segment of ridge indicates the disorientation angle, $\theta$, measured for the alloy GB underneath. Fig- 

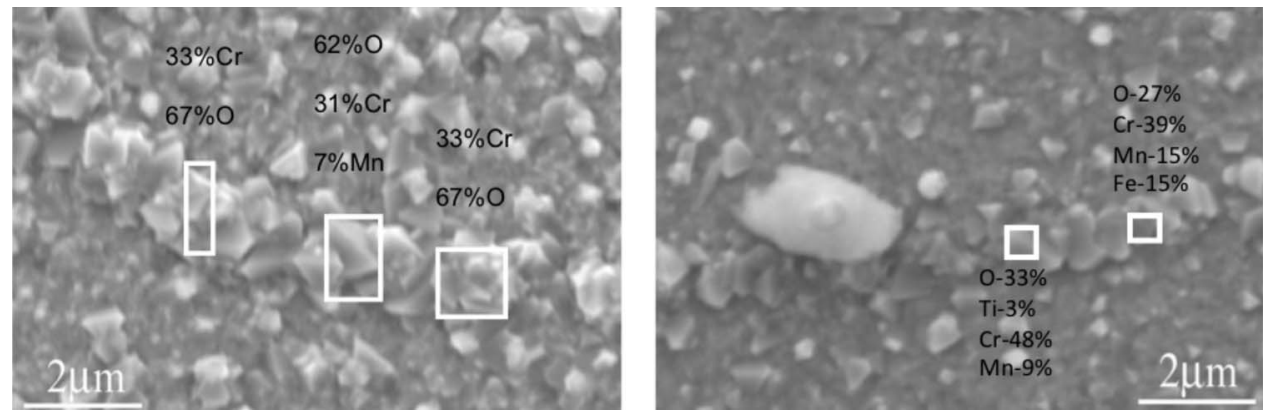

Figure 5. EDS results of the ridges on alloys $F_{1}$ and $F_{2}$, from left to right, respectively.

ure $9 \mathrm{~b}$ shows a GB map and an EBSD map, from top to bottom, of the area where the SEM image was taken. Figure 9c highlights with a square the location on the sample surface where the SEM image

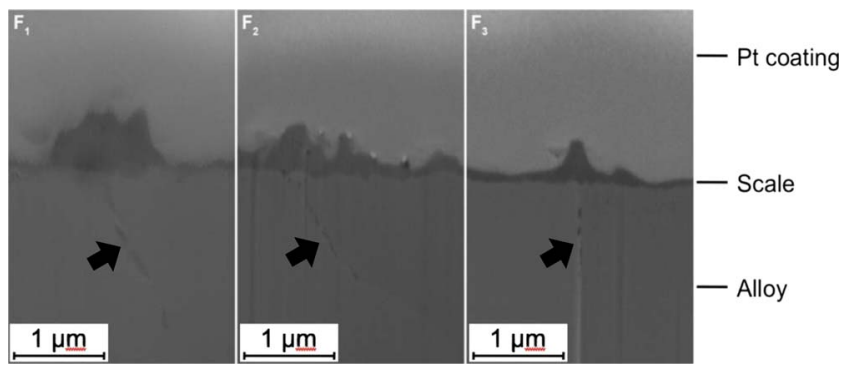

Figure 6. (Color online) FIB-milled cross sections showing scale ridges forming on top of alloy grain on group I samples. From left to right are alloys $\mathrm{F}_{1}, \mathrm{~F}_{2}$, and $\mathrm{F}_{3}$, respectively.

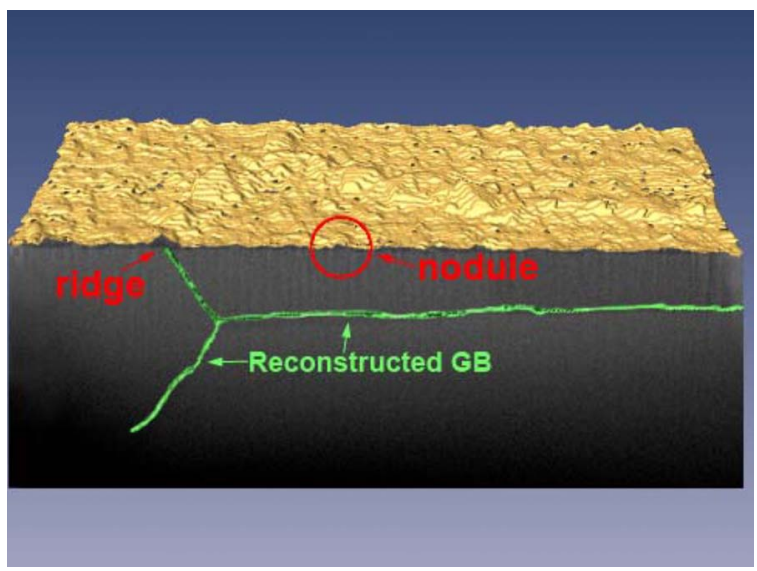

Figure 7. (Color online) Reconstructed scale layer combined with the SEM image of the cross section.
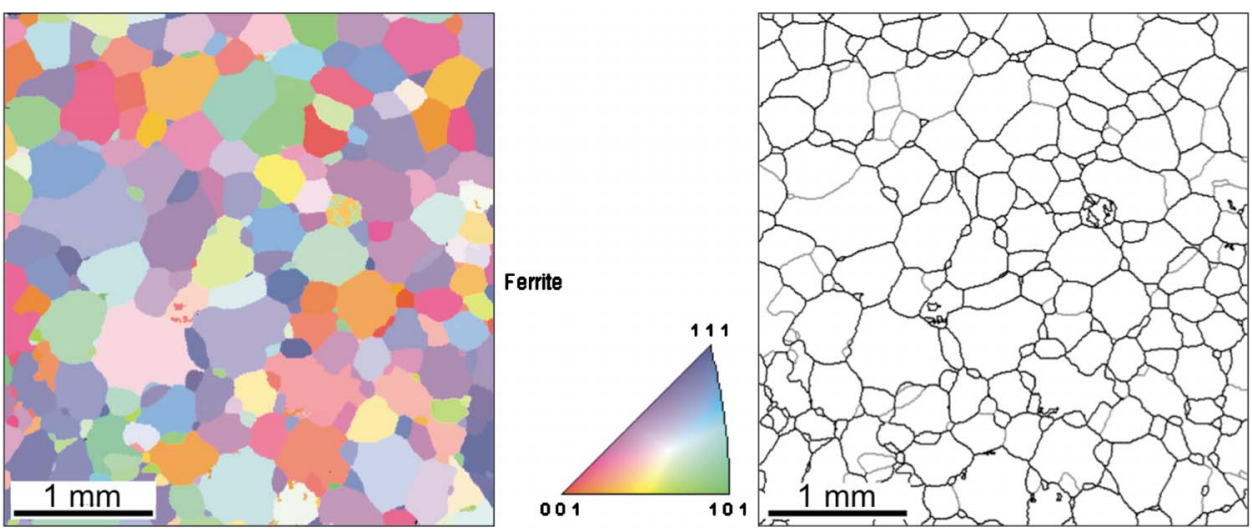

was obtained. Comparing Fig. 9a with Fig. 9b, the scale ridges clearly outlined the alloy grains beneath and matched well with the GB map. Moreover, the GB segment circled in Fig. 9b, which corresponds to a low angle boundary, has a very small ridge (circled in Fig. 9a) that has grown on top of it. In fact, almost all the alloy GBs on the sample surface with low disorientation angles (below $15^{\circ}$ ), in gray, have no or very small ridges on top of them after oxidation. There are also some outliers: Some alloy GBs with a disorientation angle larger than $15^{\circ}$ have smaller ridges on top of them as well; a few low angle boundaries have ridges that are comparable in size to high angle boundaries.

It was mentioned by Thorning and Sridhar ${ }^{13}$ that CSL boundaries were expected to have much lower diffusivities than nonspecial boundaries with the same disorientation angle. Upon examining the scale ridges in the current study, small or no ridges were found on all of the $\Sigma 3$ boundaries, with no exceptions. One example is shown in Fig. 10. Very small ridges were also found on a few $\Sigma 5$ boundary segments and even on one $\Sigma 11$ boundary segment. When the inverse of the fraction of coincident sites exceeds 13, a "normal" scale ridge formation was observed, and it seemed that it was the disorientation angle that had the most influence in terms of scale ridge formation. For example, the size of the ridge grown on top of a $\Sigma 19$ boundary was approximately the same as the other ridges formed on the nonsigma boundaries of approximately the same disorientation angle.

Figure 11 is a plot that summarizes the disorientation angles of $385 \mathrm{~GB}$ segments of the alloy surface and the sizes of scale ridges that grew from them. The ridges' size was qualitatively graded on a $0-5$ scale. Size " 5 " is the largest ridge size found on the oxidized sample surface and size " 0 " means no ridge was observable; any size in between 5 and 0 were given 1, 2, 3, or 4 accordingly, from small to large. The sizes were graded with scale ridge micrographs of the same magnification. When all the ridges were given a certain size, the disorientation angle of the alloy GB segment underneath each ridge was calculated using the OIM data analysis v5.0 software and was rounded up to the nearest integer value. The ridge sizes corresponding to the same disorientation angle were averaged, and the standard deviation of the ridge size for each disorientation angle was also calculated. The disorientation angles were then plotted

Figure 8. (Color online) Original EBSD map was cleaned up with grain dilation and then with the single (average) orientation method. (a) EBSD map of the polycrystalline alloy surface after cleaning up. (b) The inverse pole figure of ferrite. (c) Reconstructed GB map, in which gray indicates low disorientation angle $\left(<15^{\circ}\right)$ boundaries and black indicates disorientation angles larger than $15^{\circ}$. 

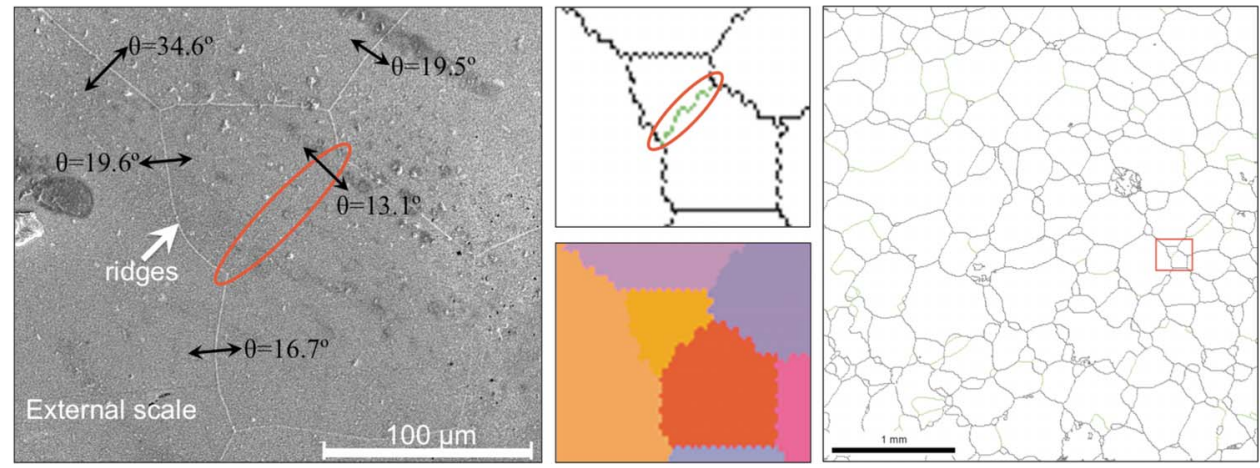

Figure 9. (Color online) (a) SEM secondary electron image of the scale ridges in a small area. (b) A GB map and an EBSD map, from top to bottom, respectively, of the area where the SEM image was taken; the disorientation angle of the circled GB segment is $13.1^{\circ}$. (c) The location on the sample surface where the SEM image was obtained.

against average ridge sizes, and the standard deviation was included in the plot as error bars (Fig. 11). The negative error bars extending below ridge size 0 were cut because a negative ridge size was never observed, and similarly, the positive error bars extending above ridge size 5 were physically meaningless.

The standard deviations were quite large in many cases due to the sample size to be averaged. The number of scale ridges graded was sometimes quite small for some of the disorientation angles, and if the scale ridge size scattered by small amounts, it would result in a large standard deviation. In contrast, in the best case, for example, there were $17 \mathrm{~GB}$ segments that had the same disorientation angle of $47^{\circ}$.

It can be seen from Fig. 11 that the low angle boundaries with disorientations in the range of $6-10^{\circ}$ have the smallest ridges grown from them with a little scatter in the size. For $10^{\circ}$ to slightly larger than $15^{\circ}$, scale ridge sizes scatter a lot, but in general, mainly small to medium sized ridges were observed. For boundaries larger than $20^{\circ}$, large ridges prevail. Exceptions were observed around 20 and $30^{\circ}$, and the ridge size dropped significantly, approaching $60^{\circ}$. In this study, most of the 59 and $60^{\circ}$ boundaries are $\Sigma 3$ boundaries.

The most important results are summarized as follows:

1. Scale ridges became visible shortly after reaching the designated temperature, and their size increased throughout the entire exposure time in this study.

2. Scale ridges were mainly composed of $\mathrm{Cr}$ oxides, but some of them were also rich in $\mathrm{Mn}$ and $\mathrm{Ti}$.

3. Scale ridge size decreased with increasing RE content in the alloy.

4. Scale ridges only grew out from underlying alloy GBs.

5. Scale ridge size differed with the disorientation angle of underlying alloy GBs.

\section{Discussion}

Diffusion in a polycrystalline material is often the result of both volume diffusion and fast-path diffusion under practical conditions. GB diffusion is a typical type of fast-path diffusion because, gener- ally, GBs are regions that are highly disordered compared to a perfect crystal and that provide a more open medium for the transportation of atoms and vacancies. The activation energy for GB diffusion in pure metals and single-phase alloys is typically half that of the activation energy for diffusion through the lattice. GB diffusion was first distinguished as having three types of diffusion kinetics in a parallel GB model by Harrison, ${ }^{8}$ and they were conventionally referred to as types A, B, and C. The quantitative criteria that distinguish these three types of GB diffusion can be found in Ref 15 .

Incorporating the experimental conditions of the present study, the kinetic regime was determined. The (tracer) diffusion coefficients of the major alloying elements of the study material can be found in Table III. The diffusion coefficients of $\mathrm{Fe}, \mathrm{Cr}$, and $\mathrm{Mn}$ at $800^{\circ} \mathrm{C}$ were therefore $4.1 \times 10^{-12}, 5.5 \times 10^{-12}$, and 9.0 $\times 10^{-12} \mathrm{~cm}^{2} \mathrm{~s}^{-1}$, respectively. Taking the actual sample grain size in this study, which is $0.182 \mathrm{~mm}$, and assuming a GB thickness of 1 $\mathrm{nm}{ }^{15}$ and an exposure time of $900 \mathrm{~s}$, the quantification criteria were calculated and are shown in Table IV, where $D$ is the volume diffusion coefficient, $t$ is the annealing time, $d$ is the grain size, and $\delta$ is the GB thickness. The term $(D t)^{1 / 2}$ is known as the mean diffusion distance.

According to Harrison's model, the diffusion kinetics of the samples in the present study fall into the type B regime, $100 \delta$ $<(D t)^{1 / 2}<d / 20$, which is mixed volume diffusion and GB diffusion. According to the mean diffusion distance criterion, at lower temperatures or shorter times, type $\mathrm{C}$ diffusion operates, which means that diffusion only occurs through GBs without a major contribution from the bulk transport. The mean diffusion distance value was calculated for the conditions of the present study and was plotted in Fig. 12. For the experimental conditions in this study, it would only take $20 \mathrm{~s}$ for the GB diffusion to transition from type $\mathrm{C}$ to type B. Although the $40 \mathrm{~s}$ heating time from room temperature to $800^{\circ} \mathrm{C}$ could prolong the type $\mathrm{C}$ regime, type $\mathrm{C}$ diffusion should still only occur for a very short time compared to the $900 \mathrm{~s}$ isothermal exposure at $800^{\circ} \mathrm{C}$. Therefore, type B diffusion likely operates for the high temperature exposure time in the present study, which is in
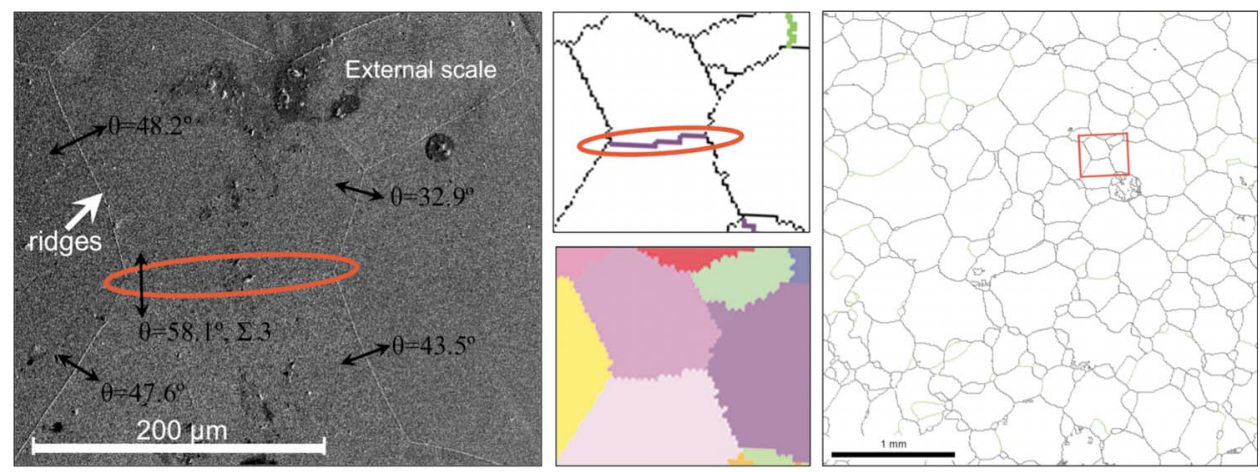

Figure 10. (Color online) (a) SEM secondary electron image of the scale ridge in a small area. (b) A GB map and an EBSD map, from top to bottom, respectively, of the area where the SEM image was taken. The circled GB segment is a $\Sigma 3$ boundary. (c) The location on the sample surface where the SEM image was obtained. 


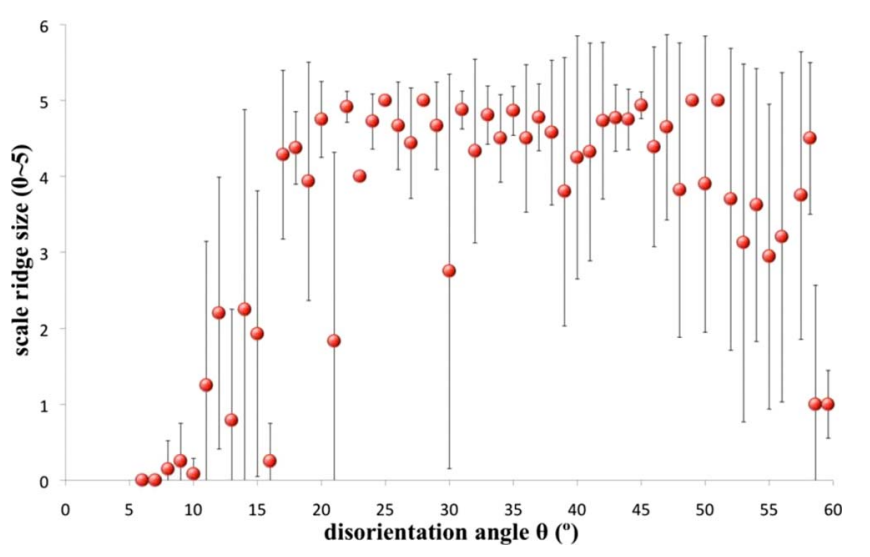

Figure 11. (Color online) The disorientation angles were plotted against average ridge sizes with the standard deviation included in the plot as error bars.

accordance with the real-time observation in CSLM. The scale ridges appeared shortly after the gas-phase mass-transfer process started and before scale growth began in the interiors of the grains. As the exposure continued, the ridges grew larger along with the scales growing within the grains, which could be considered as a transition from type $\mathrm{C}$ to type $\mathrm{B}$ kinetics. The quantitative criteria are not applicable to long-term oxidation because the scale growth is controlled by the diffusion of metal or oxygen species through the oxide scale, not the metal matrix.

With the help of real-time CSLM observation and FIB cross sections of the scale ridges, the formation of scale ridges can be portrayed as follows.

Scale ridges appear shortly after the alloy has been exposed to an oxidizing atmosphere at a given high temperature because of a preferential fast diffusion path: GBs. As the height of the ridges increase, however, the diffusion distance in the protrusions increases as well, which consequently slows down the ridge growth rate. When the height of the ridges becomes large enough, the growth at the base of the ridges becomes faster than the growth of ridge height (Fig. 13a and b). ${ }^{16} \mathrm{Next}$, along with the accumulation of oxides at the base of the ridges, the scale growth rate in the interiors of the grains should gradually catch up with ridge growth along alloy GBs, and the scale ridges would be expected to finally flatten out (see the schematics in Fig. 13).

Before discussing the relation between scale ridge size and the characteristics of alloy GBs, it must be shown that the heterogeneity of alloy GBs intersecting the surface is important. This is analyzed by employing the "diffusion distance" term and by a GB diffusion model proposed by Thorning and Sridhar. ${ }^{13}$ The longest distance that Mn atoms can diffuse within a given time (diffusion distance) was calculated because $\mathrm{Mn}$ is the element with the largest bulk diffusion coefficient. Because $D_{\mathrm{Mn}}=9.0 \times 10^{-12} \mathrm{~cm}^{2} \mathrm{~s}^{-1}$, the diffusion distance for the exposure time in this study gives $\left(D_{\text {Mn,lattice }} t\right)^{1 / 2}=9 \times 10^{-5} \mathrm{~cm}$. The diffusion distance does not exceed the grain size in the current study, which means that only the first layer of grains are pertinent for the transport process and that the surface GBs may be acting like "bottlenecks" for outward dif-

\begin{tabular}{|c|c|c|c|}
\hline \multirow[b]{2}{*}{ Criteria } & \multicolumn{3}{|c|}{ Elements } \\
\hline & $\begin{array}{c}\mathrm{Cr} \text { in } \mathrm{Fe}(\mathrm{BCC}) \\
(\mathrm{cm})\end{array}$ & $\begin{array}{c}\mathrm{Fe} \text { in } \mathrm{Fe}(\mathrm{BCC}) \\
(\mathrm{cm})\end{array}$ & $\begin{array}{c}\text { Mn in } \mathrm{Fe}(\mathrm{BCC}) \\
(\mathrm{cm})\end{array}$ \\
\hline $\begin{array}{l}(D t)^{1 / 2} \\
100 \delta \\
d / 20\end{array}$ & $6.788 \times 10^{-5}$ & $\begin{array}{c}6.106 \times 10^{-5} \\
1 \times 10^{-5} \\
9.1 \times 10^{-4}\end{array}$ & $9.000 \times 10^{-5}$ \\
\hline
\end{tabular}

fusing species through them, and their characteristics greatly determine the diffusivity that governs the kinetics of the growth of ridges.

From the results in the current study (Fig. 9-11), apparently not all the GBs intersecting the scale/alloy interface are associated with oxide ridges and that there is a variation in the sizes of the ridges. Furthermore, the fact that a correlation between scale ridge sizes and GB characteristics was revealed suggested that GB diffusivity must be dependent upon GB structure and/or characteristics.

Although there have been many experimental investigations that indicate that GB diffusivity is dependent on the misorientation between grains, the relation between the GB structure and the corresponding diffusivity has only been studied for a limited number of well-defined boundaries. ${ }^{14,15,17}$

The first quantitative study on GB diffusion was carried out by Turnbull and Hoffman in 1954. ${ }^{17}$ They found in a tilt GB of silver bicrystals that $\delta D_{\mathrm{b}}$ increased with increasing tilt misorientation $(\theta)$, following a $\sin (\theta / 2)$ variation for small $\theta$. Subsequent work also revealed that GBs with large misorientation angles exhibited cusps on a $\delta D_{\mathrm{b}}$ vs $\theta$ plot at angles corresponding to those of sigma boundaries (CSL misorientation angles). ${ }^{15}$ In the present study, similar trends were found as those mentioned in Ref. 17 and 15.

Recent studies on GB diffusivity ${ }^{18-21}$ are significantly scattered in terms of GB diffusion coefficients measured in several metal and oxide systems. Perhaps the variation in the structures of the GBs studied by these researchers could be one cause of the data scattering because it was observed in this study that GBs with different characteristics had different diffusivities.

However, few studies have been dedicated to relationships between scale ridge formation and GB characteristics. Thorning and Sridhar ${ }^{13}$ found that intergranular oxidation at high angle alloy GBs had a pronounced effect on initial oxide morphology on the alloy surface, whereas no such effect was observed at low angle GBs in a transformation-induced plasticity steel. They also investigated the scale ridges that were associated with alloy GBs, but no unique correlation between the GB disorientation angle and the observed heterogeneities in oxide ridge evolution was found.

The GB misorientation was described in this study as the disorientation, and the major types of GBs in our study were low angle nonspecial GBs, high angle nonspecial GBs, and CSL boundaries. A clear relation between the scale ridge size and the alloy GB disorientation angle was revealed: The scale ridge size increased with increasing disorientation angle, as shown in Fig. 11. Moreover, CSL boundaries with low sigma numbers, i.e., $\Sigma 3$ and $\Sigma 5$ boundaries, possess low diffusivity. This was indicated in Fig. 11 as a cusplike drop in scale ridge size at the corresponding angles for $\Sigma 3\left(60^{\circ} /\right.$ $[111])$ and $\Sigma 5\left(37^{\circ} /[100]\right)$ boundaries. In Fig. 10, a specific example

Table III. Pertinent (tracer) diffusion coefficient data for substitutional elements.

\begin{tabular}{lccccc} 
Alloy & Element & $\begin{array}{c}\text { Temperature } \\
(\mathrm{K})\end{array}$ & $\begin{array}{c}E \\
\left(\mathrm{~kJ} \mathrm{~mol}^{-1}\right)\end{array}$ & $\begin{array}{c}D_{\mathrm{o}} \\
\left(\mathrm{cm}^{2} \mathrm{~s}^{-1}\right)\end{array}$ & Reference $^{-1}$ \\
\hline Fe body-centered cubic (BCC) & $\mathrm{Fe}$ & $1043-1809$ & 240 & 2.0 & 9 \\
Fe (BCC) & $\mathrm{Cr}$ & $1048-1971$ & 238.8 & 2.33 & 10 \\
Fe (BCC) & $\mathrm{Mn}$ & $1063-1173$ & 224.5 & 0.756
\end{tabular}




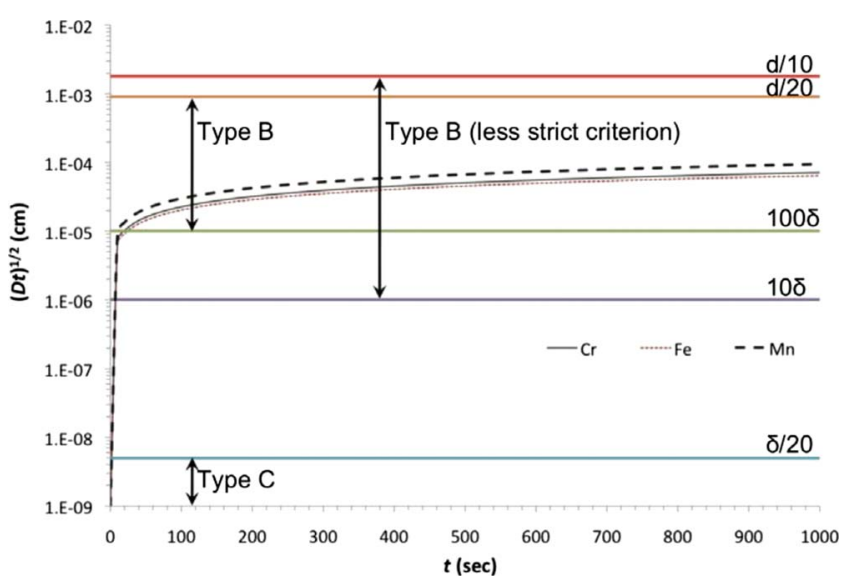

Figure 12. (Color online) The mean diffusion distance calculated for $\mathrm{Cr}$ and $\mathrm{Fe}$ in a BCC matrix of iron is plotted vs time in an exposure time of $1000 \mathrm{~s}$ at $800^{\circ} \mathrm{C}$.

of such a case is shown. It seems reasonable that $\Sigma 3$ and $\Sigma 5$ boundaries are associated with better atomic fit at the GB interface, which implies that a smaller amount of vacancies and/or less complicated dislocation structures would be required within the GBs themselves. Therefore, the expected increase in the GB diffusion coefficient with a disorientation angle may not be prevalent in these cases.

The effect of GB misorientation on GB diffusivity, as shown in Fig. 11, is discussed here in terms of diffusion coefficient and/or diffusivity. GB diffusivity at a given temperature $T$ and the product $\delta D_{\mathrm{GB}}$ is composed of a pre-exponential factor, $D_{\mathrm{oGB}}$ (assumed to be independent of pressure and temperature) and an exponential term including activation energy and temperature, which is similar to the diffusion coefficient of volume diffusion. The GB thickness is $\delta$, and $D_{\mathrm{GB}}$ is the GB diffusion coefficient.
The $D_{\mathrm{o}}$ for volume diffusion is more or less constant at a given temperature $^{22}$ from which it can be deduced that $D_{\mathrm{oGB}}$, similar to $D_{\mathrm{o}}$, can be taken as a constant for a solid of a given structure and bond type, ${ }^{22}$ assuming the same vacancy diffusion mechanism operates for both volume diffusion and GB diffusion. In other words, the structure of the GB, i.e., misorientation, has little influence on $D_{\mathrm{oGB}}$.

However, the exponential term in $D_{\mathrm{GB}}$ could have a strong dependence on the disorientation angle and on the GB structure. Differences in free space at different boundary interfaces should result in a difference in diffusion activation energy and in activation volume. The free space at a small-angle boundary can be expected to be smaller than that of a large-angle boundary, and particularly for some $\Sigma 3$ boundaries, the free space at the boundary should also be very small. Therefore, although the volume of a boundary vacancy formation is the same for a small-angle or CSL boundary as that for a large-angle nonspecial boundary, when a diffusive jump occurs in a small-angle or CSL boundary, the free volume that is associated permanently with the boundary makes the jump possible with more local dilation than that required within a high angle nonspecial boundary plane; therefore, the volume needed for the migration of a boundary vacancy, as well, should be larger than that of a vacancy at a high angle nonspecial boundary. This would result in a higher activation energy and an activation volume that give rise to a decrease in the GB diffusion coefficient.

The GB free space is also associated with GB thickness $\delta$, although the effect is not as prominent as the exponential terms: activation energy and volume. The $\delta$ of a small-angle boundary $\left(5-10^{\circ}\right)$ is smaller than that of boundaries larger than $20^{\circ}$. It is also noticeable that for boundaries above $20^{\circ}$, the scale ridge size will not vary that much, unless special (CSL) boundaries are present. This is in accordance with the fact that the boundary thickness and the degree of disorder at the GB interface of boundaries larger than $20^{\circ}$ do not vary much and therefore do not influence diffusivity much. However, low sigma number CSL boundaries, despite their large disorientation angles, e.g., $60^{\circ}$ for $\Sigma 3$ boundary, have much less disordered boundary interfaces than nonspecial boundaries with the same

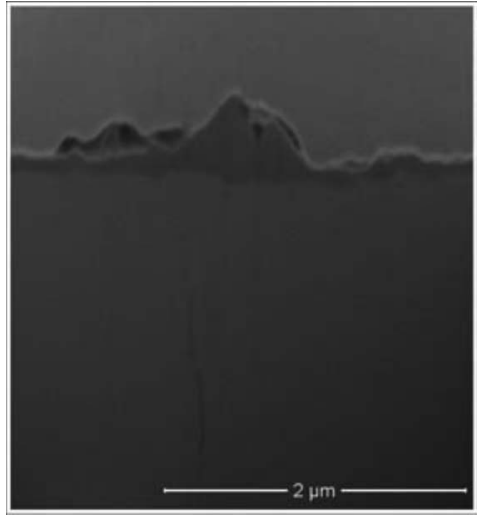

a)

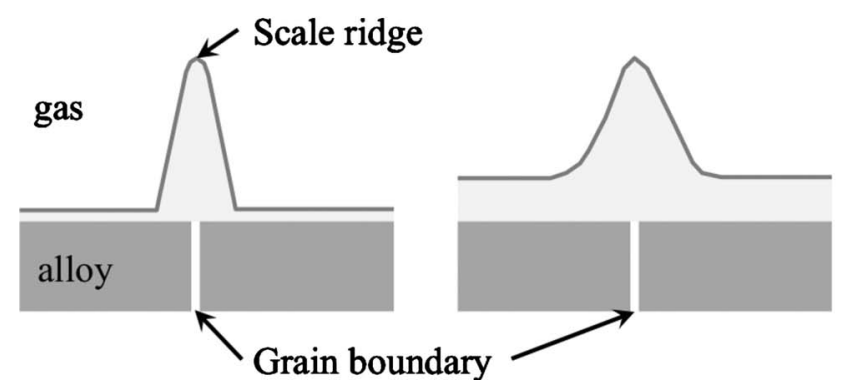

c)

d)

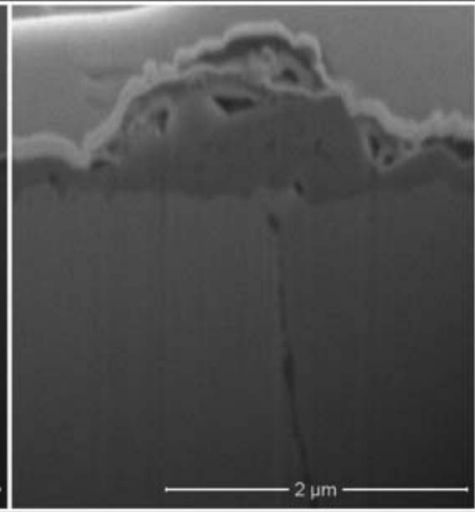

b)

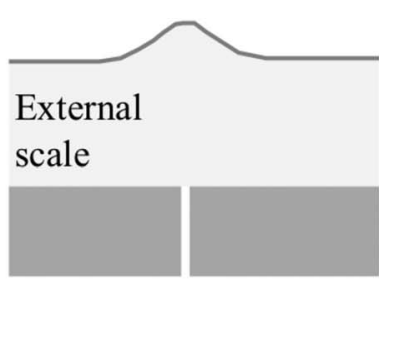

e) 

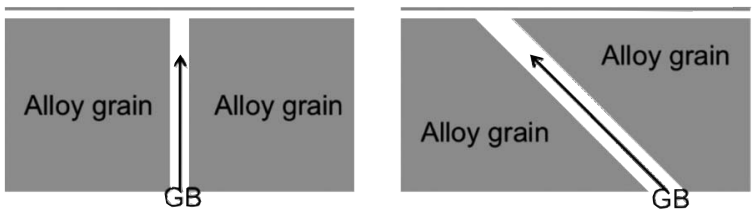

Figure 14. Schematic of the inclined GB to free surface.

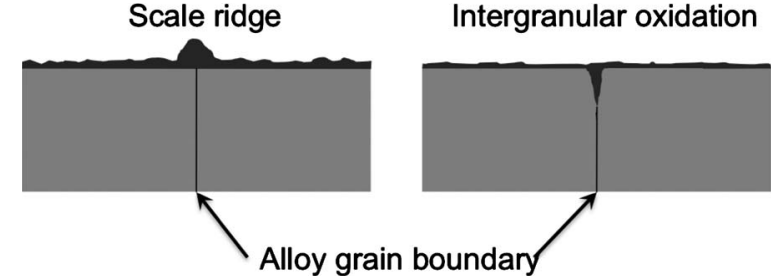

Figure 15. Schematic of scale ridge and intergranular oxidation.

Fig. 15, formed along GBs, then after a while diffusion would have no longer been solely under the effect of the misorientation of the alloy GBs but also the diffusion through the internally formed oxides or along the internal oxide/alloy interface. Therefore, the extent and nature of the intergranular oxides formed along GBs must also be taken into account with relevant data available.

In the present study, the oxide nodules that formed the ridges were mainly $\mathrm{Cr}$ oxides, but some scale ridges were also rich in Mn There has been speculation that $\mathrm{Mn}$ might have already segregated at GBs during thermal annealing because $\mathrm{Mn}$ is known as a strong GB segregant in steels, ${ }^{26-28}$ and therefore the Mn source at GBs is larger than that within the grains, which gives rise to its enrichment in the scale ridges. ${ }^{13}$ However, the diffusion coefficients calculated in the previous section may also provide insight. The volume diffusion coefficient for $\mathrm{Mn}$ at $800^{\circ} \mathrm{C}$ is almost twice that of $\mathrm{Fe}$ or $\mathrm{Cr}$. Because the GB diffusion coefficient is normally orders of magnitudes larger than the volume diffusion coefficient, with the same GB thickness assumed, the difference between volume diffusivity and that of the GB is significantly larger for $\mathrm{Mn}$ than for $\mathrm{Fe}$ or $\mathrm{Cr}$. In other words, the difference in diffusivity is much more amplified at the GB for Mn, and this would possibly lead to the observed enrichment of $\mathrm{Mn}$ in the scale ridges. However, the two possibilities stated here do not preclude each other. It is possible that the segregation of $\mathrm{Mn}$ before oxidation and the faster Mn diffusion at GB than $\mathrm{Cr}$ and $\mathrm{Fe}$ are both reasons of $\mathrm{Mn}$ enrichment in the ridges.

The alloy in this study is considered as a candidate SOFC interconnector material, so both disadvantages and advantages may arise from scale ridge formation during service as an interconnector.

The mechanical properties of the scale may be jeopardized by ridge formation and heterogeneity of the scale. Although scale fracture typically occurs in steady-state scales after hundreds or even thousands of hours of oxidation, on which barely any ridges would be visible, experimental evidence (Fig. 6 and 13b) revealed that scale ridge formation during the early transient oxidation sometimes roughened the metal/scale interface. The roughened interface became a potential stress origin during cooling. ${ }^{29}$ Moreover, variation in the thickness of the scale may result in the accumulation of stress within the scale, ${ }^{29}$ especially during heating or cooling, and later, such force can create cracks in the scale that leads to spalling of the scale and, consequently, to a rapid loss of base alloy.

Electrical contact and electrical resistance is important for interconnectors. Electrical resistance does not likely change because of ridge formation according to the electrical resistance measured during a long-term high temperature exposure. ${ }^{30}$ The overall electrical resistance varies only a little in the first $150 \mathrm{~h}$, and by that time, the ridges can be expected to flatten out completely. Conversely, electrical contact may be altered by ridge formation by changing the area of the contact if no other aspect is considered. In this case, if the scale grows locally that changes the contact area, this changes the area specific resistance.

If $\mathrm{RE}$ additions can reduce the tendency to form ridges, then the heterogeneity of the scale can be reduced, and therefore, better mechanical properties of the scale can be expected such that spalling and buckling of the scale can be alleviated.

The Mn enrichment found in some ridges is advantageous with respect to spinel formation. Manganese is added into a Crofer 22- 
type stainless steel aiming at alleviating the $\mathrm{Cr}$ poisoning issue associated with volatile species of $\mathrm{Cr}$ oxides. If more $\mathrm{Mn}$ could be delivered to the scale layer through ridge formation, then more spinel can form and therefore help stabilize the volatile $\mathrm{Cr}$ oxide.

\section{Conclusions}

Scale ridge growth on the surface of alloys similar to a Crofer 22 stainless steel was studied with live oxidation video recorded by using CSLM at $800^{\circ} \mathrm{C}$ in dry air. Subsequently, the scale ridges on the samples' surfaces were characterized using SEM and EDS. Some ridges were rich in $\mathrm{Mn}$.

$\mathrm{RE}$ addition can reduce the tendency of ridge formation. The size of the ridges decreased with increasing RE content in the alloy, which was probably due to more segregation of impurities at alloy GBs resulting from RE addition.

The SEM imaging of the scale ridges combined with the EBSD mapping of underlying alloy grains revealed a relation between the ridge size and the misorientation of the alloy GB underneath. Among nonspecial GBs, small ridges usually corresponded to small angle boundaries beneath, and large ridges corresponded to large angle boundaries. Sigma 3 and 5 boundaries also had very small ridges on top of them despite their large disorientation angles.

If GB diffusivity can be represented by the scale ridge size, then the GB diffusivity is small when the activation energy and/or the activation volume is large, which is the case of a less disordered GB interface (either a small angle boundary or a CSL boundary).

\section{Acknowledgments}

This technical effort was performed in support of the NETL's ongoing research in the study of the effects of RE elements on the high temperature oxidation of stainless steels under the RDS contract DE-AC26-04NT41817. The authors acknowledge the use of MRSEC facilities supported by NSF grant DMR-0520425 and the excellent technical support on electron microscopy of Tom Nuhfer. The authors also thank Professor Gregory S. Rohrer and Jia Li for their very helpful comments and discussions on EBSD techniques and GB topics.

Carnegie Mellon University assisted in meeting the publication costs of this article.

\section{References}

1. N. Q. Minh and T. Takahashi, Science and Technology of Ceramic Fuel Cells, Elsevier Science \& Technology, New York (1995).

2. W. J. Quadakkers, J. Piron-Abellan, V. Shemet, and L. Singheiser, Mater. High Temp., 20, 115 (2003).

3. P. Y. Hou, K. Huang, W. T. Bakker, S. C. Singhal, and M. Dokiya, Editors, PV 99-19, p. 737, The Electrochemical Society Proceedings Series, Pennington, NJ (1999).

4. N. Bricks, G. H. Meier, and F. S. Pettit, High Temperature Oxidation of Metals, 2nd ed., Cambridge University Press, Cambridge, U.K. (2006).

5. R. Hojda, W. Heimann, and W. J. Quakakkers, in ThyssenKrupp Techforum, ThyssenKrupp VDM (2003).

6. B. Chattopadhyay and G. C. Wood, Oxid. Met., 2, 373 (1970)

7. L. M. Fernandez Diaz, J. Zhu, G. R. Holcomb, P. D. Jablonski, D. E. Alman, and S. Sridhar, Defect Diffus. Forum, 283-286, 425 (2009).

8. L. G. Harrison, Trans. Faraday Soc., 57, 1191 (1961).

9. H. Oikawa, Tech. Rep., Tohoku Univ., 46, 67 (1982).

10. A. M. Huntz, M. Aucouturier, and P. Lacombe, C. R. Acad. Sci. URSS, C265, 554 (1947).

11. K. Nohara and K. Hirano, in Proceedings of the International Conference on Science and Technology of Iron and Steels, Suppl. to Trans. Iron Steel Inst. Japan, The Iron and Steel Institute of Japan, p. 1267 (1971).

12. S. I. Wright and R. J. Larsen, J. Microsc., 205, 245 (2002).

13. C. Thorning and S. Sridhar, Philos. Mag., 87, 3479 (2007).

14. A. P. Sutton and R. W. Balluffi, Interfaces in Crystalline Materials, Oxford University Press, Oxford (1995).

15. I. Kaur, Y. Mishin, and W. Gust, Fundamentals of Grain and Interphase Boundary Diffusion, 3rd ed., John Wiley \& Sons, New York (1995).

16. H. S. Hsu and G. J. Yurek, Oxid. Met., 17, 55 (1982).

17. D. Turnbull and R. E. Hoffman, Acta Metall., 2, 419 (1954).

18. W. Preis and W. Sitte, Solid State Ionics, 179, 765 (2008).

19. J. S. Dohie, J. R. Cahoon, and W. F. Caley, J. Phase Equilib. Diffus., 28, 322 (2007).

20. T. Nakagawa, I. Sakaguchi, N. Shibata, K. Matsunaga, T. Mizoguchi, T. Yamamoto, H. Haneda, and Y. Ikuhara, Acta Mater, 55, 6627 (2007).

21. A. Inoue, H. Nitta, and Y. Iijima, Acta Mater, 55, 5910 (2007).

22. A. M. Brown and M. F. Ashby, Acta Metall., 28, 1085 (1980).

23. B. A. Pint, Oxid. Met., 45, 1 (1996).

24. T. Horita, H. Kishimoto, K. Yamaji, Y. Xiong, N. Sakai, M. E. Brito, and H. Yokokawa, Solid State Ionics, 179, 1320 (2008).

25. T. Skidmore, R. G. Buchheit, and M. C. Juhas, Scr. Mater, 50, 873 (2004).

26. W. Wei and H. J. Grabke, Corros. Sci., 26, 223 (1986).

27. P. Maier and R. G. Faulkner, Mater. Charact., 51, 49 (2003).

28. P. Maier, R. G. Faulkner, P. Spellward, and J. R. Cowan, Mater. Sci. Technol., 17, 1377 (2001)

29. A. G. Evans and R. M. Cannon, Mater. Forum, 43, 243 (1989).

30. Crofer 22 APU, Material Data Sheet no. 4046, June 2008 ed., ThyssenKrupp VDM. 